\title{
Wireless Multichannel Multipoint Broadcast Service for Mobile Stations
}

\author{
B Homer Benny, K Chaitanya Deepti, P Ratna Kumar \\ Department of Computer Science and Engineering, Sir CRR College of Engineering, Eluru.
}

\begin{abstract}
In wireless Multicast Broadcast Service (MBS), the common channel is used to multicast the MBS content to the Mobile Stations (MSs) on the MBS calls within the coverage area of a Base Station (BS), which causes interference to the dedicated channels serving the traditional calls, and degrades the system capacity. The MBS zone technology is proposed in Mobile Communications Network (MCN) standards to improve system capacity and reduce the handoff delay for the wireless MBS calls. In the MBS zone technology, a group of BSs form an MBS zone, where the macro diversity is applied in the MS, the BSs synchronize to transmit the MBS content on the same common channel, interference caused by the common channel is reduced, and the MBS MSs need not perform handoff while moving between the BSs in the same MBS zone. However, when there is no MBS MS in a BS with the MBS zone technology, the transmission on the common channel wastes the bandwidth of the BS. It is an important issue to determine the condition for the MBS Controller (MBSC) to enable the MBS zone technology by considering the Quality of Services (QoS) for traditional calls and MBS calls are used to reduce the dependency over the common channel and also it is going to reduce the delay over the network. By enabling Dynamic Channel Allocation (DCA) and Enhance Dynamic Channel Allocation (EDCA) we are going to overcome these problems.
\end{abstract}

Keywords: Multicast Broadcast Service (MBS), Mobile Communications Network (MCN), Improve Dynamic Channel Allocation (DCA), Enhance Dynamic Channel Allocation (EDCA)

\section{Introduction}

The existing system is usually called a time-free asynchronous distributed system pron e to process crashes. In these systems, a system designer can only assume an upper bound on the number of processes that can crash and, consequently, design protocols relying on the assumption that at least processes are alive. The protocol has no means to know whether a given process is alive or not. The common channel is used to multicast the MBS content to the Mobile Stations (MSs) on the MBS calls within the coverage area of a Base Station (BS), which causes interference to the dedicated channels serving the traditional calls, and degrades the system capacity.

Figure 1 illustrates the general MCN architecture with the MBS zone technology. The MBS Controller (MBSC; Figure 1 (3)) accommodates the functionalities including the MBS zone management, service announcement, membership management, security management, session management, session transmission, multicast connection identifier and IP address management. The MBS Server (Figure 1 (1)) is the MBS content provider. The Multicast Router (Figure 1 (2)) performs multicast routing for the MBS content in the IP network.

Two types of logical channels (including common channel and dedicated channel) are used to transmit the MBS content. Figure 2 illustrates the channel pool consisting of common channels and dedicated channels. The transmission of a dedicated channel is point-to-point between the Base Station (BS) and the Mobile Station (MS), which serves either an MBS call or a traditional call.

The transmission of a common channel is point-to-multipoint between the BS and a group of MSs. The common channel can be used to multicast the MBS content to all MSs subscribing the same MBS in the coverage area of the BS (known as the cell). Compared with the dedicated channel, there is no associated control channel for the common channel to report the channel quality of the MS. To cover the whole cell, the transmission power of a common channel should be large enough. Therefore, the usage of a common channel may result in higher interference to the dedicated channels. It is recommended to use a common channel to 
deliver the MBS content while the number of MSs listening to the MBS content is sufficiently large.

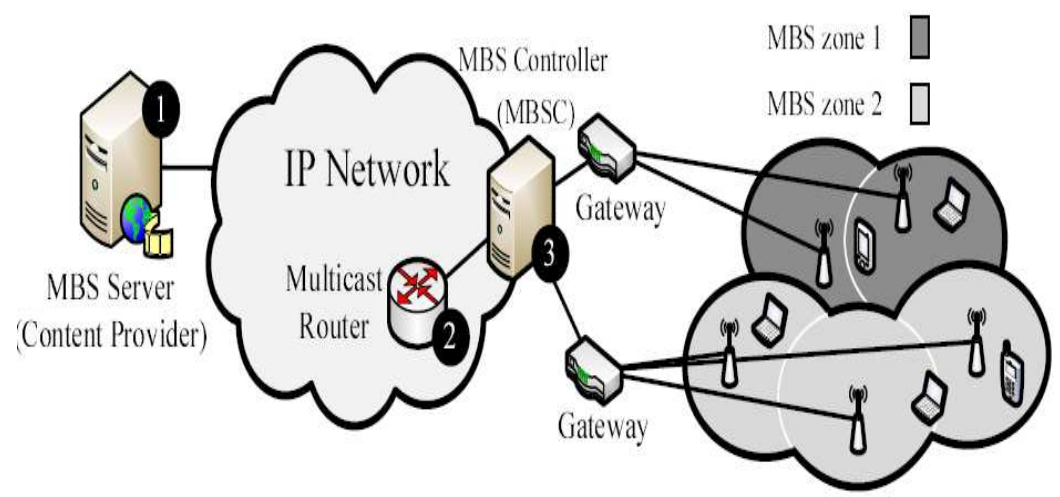

Figure 1. The MCN architecture with the MBS zone technology

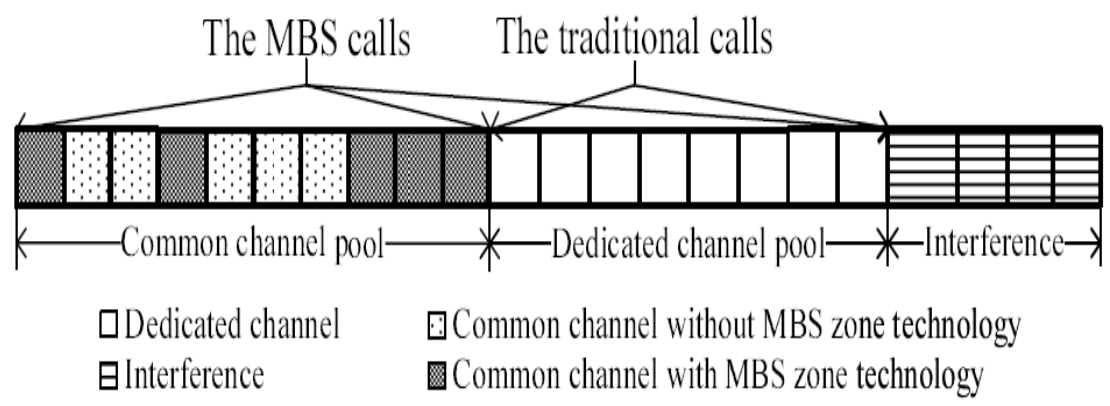

Figure 2. The logical channelpool of a BS

The Purpose of this application is to reduce the dependency over the common channel and also it is going to reduce the delay over the network. By enabling Dynamic Channel Allocation (DCA) and Enhance Dynamic Channel Allocation (EDCA) we are going to overcome these problems. Dynamic Channel Allocation (DCA) and Enhanced Dynamic Channel Allocation (EDCA), for more flexible channel allocation in the MBS network. In Dynamic Channel Allocation (DCA), the MBS network determines whether the MBS zone technology should be enabled or disabled when an MS joins/leaves the MBS service. Scheme EDCA enhances DCA by also considering the behavior of the traditional call.

To improve the system capacity and reduce the handoff delay for wireless Multicast and Broadcast Service (MBS), The MBS zone technology is being proposed in several Mobile Communications Network (MCN). In the MBS zone technology, an MBS zone consists of a group of BSs synchronized to transmit the same MBS content using the same common channel. The transmission power required for a common channel with the MBS zone technology is less than that without the MBS zone technology due to the macro diversity, and the interference caused by the common channels is reduced. Suppose that it damages $d z$ and $d c$ (where $d z \leq$ $d c$ ) dedicated channels to turn on a common channel with the MBS zone technology and without the MBS zone technology, respectively.

Dynamic Channel Allocation (DCA) and Enhanced Dynamic Channel Allocation (EDCA), are more flexible channel allocation in the MBS network. In Dynamic Channel Allocation (DCA), the MBS network determines whether the MBS zone technology should be enabled or disabled when an MS joins/leaves the MBS service. Scheme EDCA enhances DCA by also considering the behaviour of the traditional call. The proposed schemes are very practical to be implemented in the MBS network. 


\section{Research}

Literature Review is the most important step in software development process. Before developing the tool it is necessary to determine the time factor, economy and company strength. Once these things are satisfied, then next steps are to determine which operating system and language can be used for developing the tool. Once the programmers start building the tool the programmers need lot of external support. This support can be obtained from senior programmers, from book or from websites. Before building the system the above consideration are taken into account for developing the proposed system.

In this section, we propose two Dynamic Channel Allocation schemes, Dynamic Channel Allocation (DCA) and Enhance Dynamic Channel Allocation (EDCA), for the MBS network. In Dynamic Channel Allocation (DCA), the Multicast Broadcast Service Controller (MBSC) attempts to activate the MBS zone technology if the number of MBS calls in all cells (belonging to the same MBS zone) is equal or larger than a pre-defined $\theta$. When the MBS zone technology is disabled, DCA turns on a common channel to serve the MBS calls if the number of MBS calls in the cell is larger than dc. The EDCA enhances DCA by putting the behavior of the traditional call into consideration. When a traditional call arrives in a cell where there is no free dedicated channel, EDCA attempts to have more dedicated channels by activating or deactivating the MBS zone technology.

In Dynamic Channel Allocation (DCA), the activation of the common channel with/without the MBS zone technology is determined after the MBSC checks the channel status of all cells in an MBS zone, which introduces signalling message exchange overhead. Similar to Dynamic Channel Allocation (DCA), not only for the activation of the common channel but also for the traditional call requests, EDCA introduces higher signalling message exchange overhead than Dynamic Channel Allocation (DCA) does. Dynamic Channel Allocation (DCA) and EDCA may have better system capacity, but more signalling overhead.

\section{Methodology}

The data flow diagram is used for classifying system requirements to major transformation that will become programs in system design. This is starting point of the design phase that functionally decomposes the required specifications down to the lower level of details. It consists of a series of bubbles joined together by lines.

Bubbles: Represent the data transformations.

Lines: Represents the logic flow of data.

NOTATIONS USED IN DATA FLOW DIAGRAMS:

The logic dataflow diagrams 4,5 , and 6 can be drawn using only four simple notations i.e., special symbols or icons and the annotation that associates them with a specific system.

\section{Element References \\ Data Flow Process \\ Process}

Data Store

Source or Sink
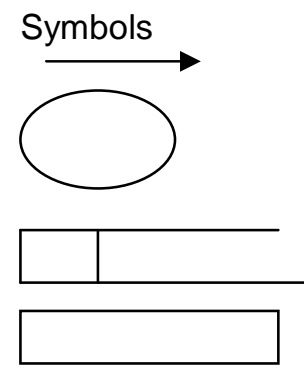

\subsection{Data Flow Diagram}

Figure 3-6 show the data flow diagram of this research. 


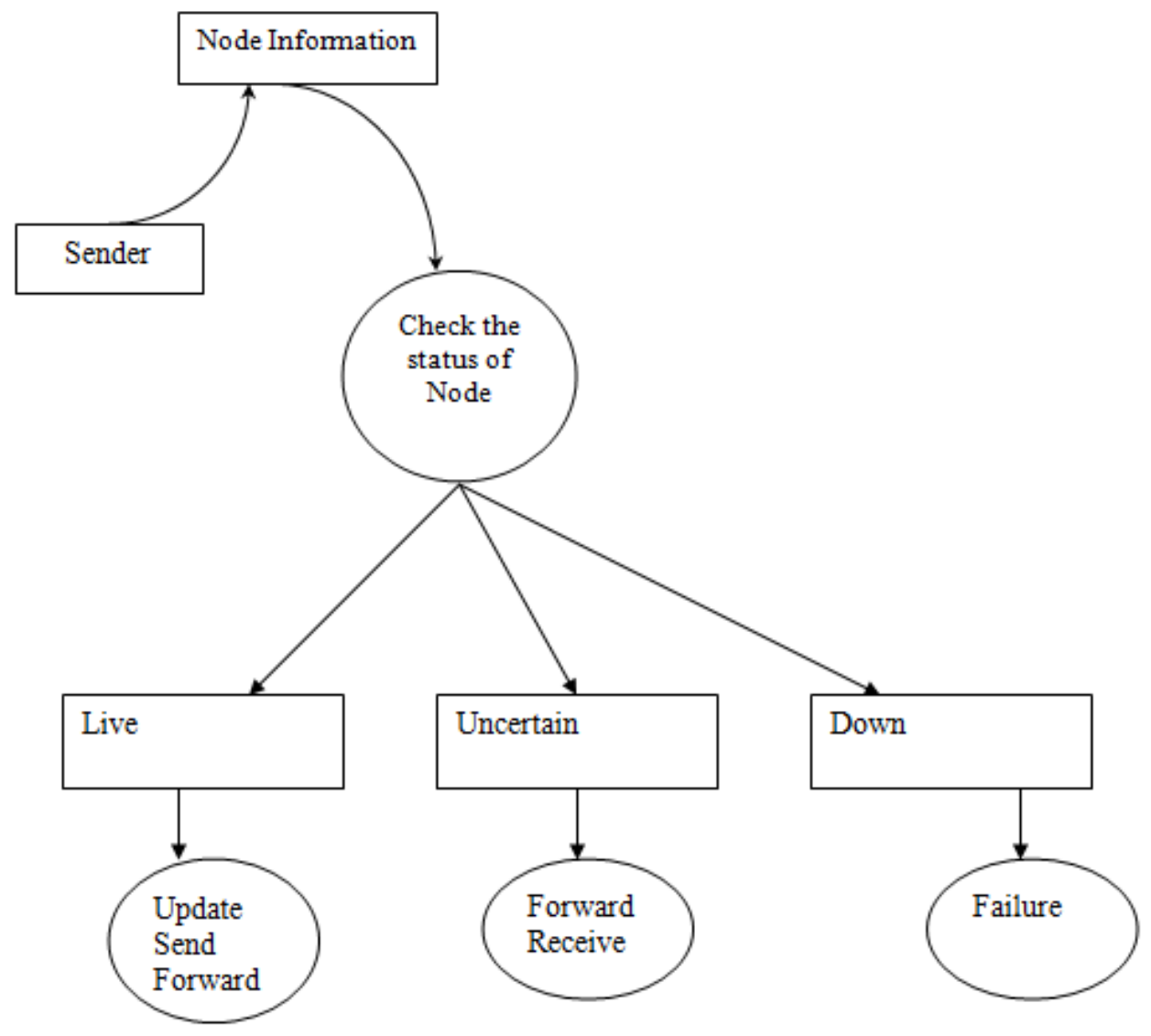

Figure 3. Identify the status of node

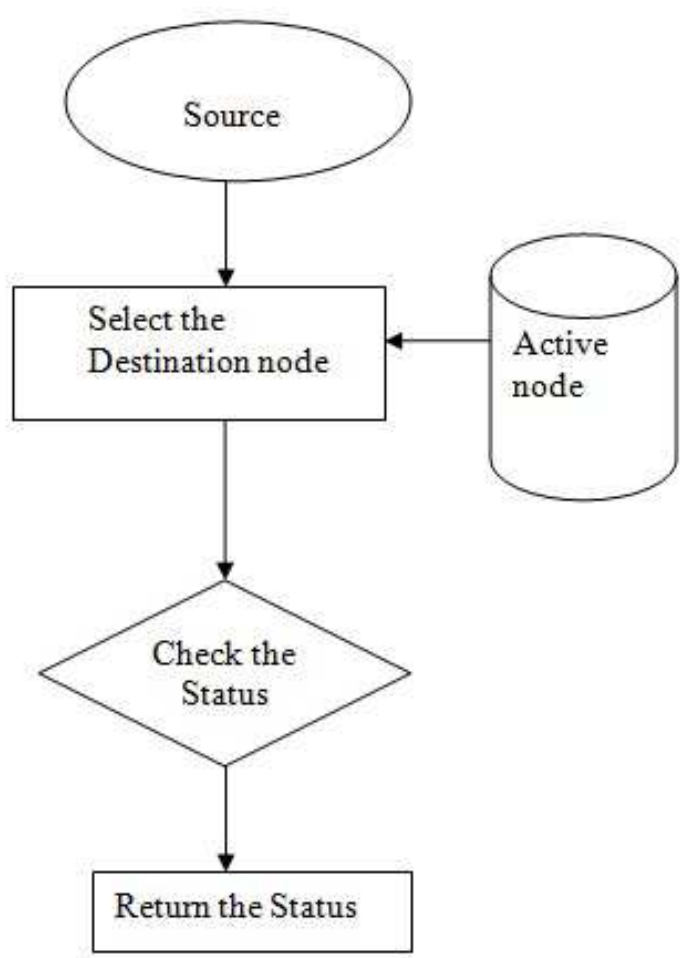

Figure 4. Status of the node 


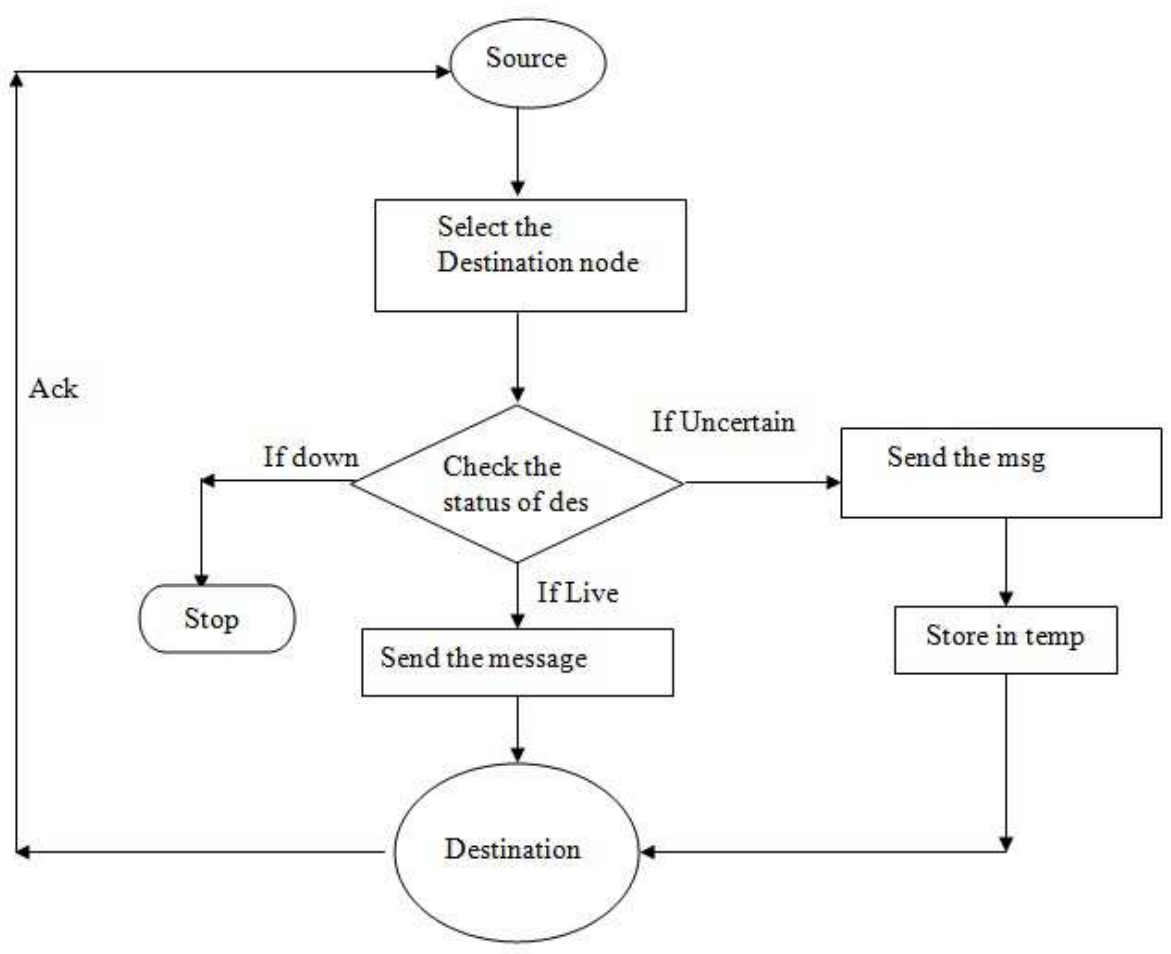

Figure 5. Message Transmission

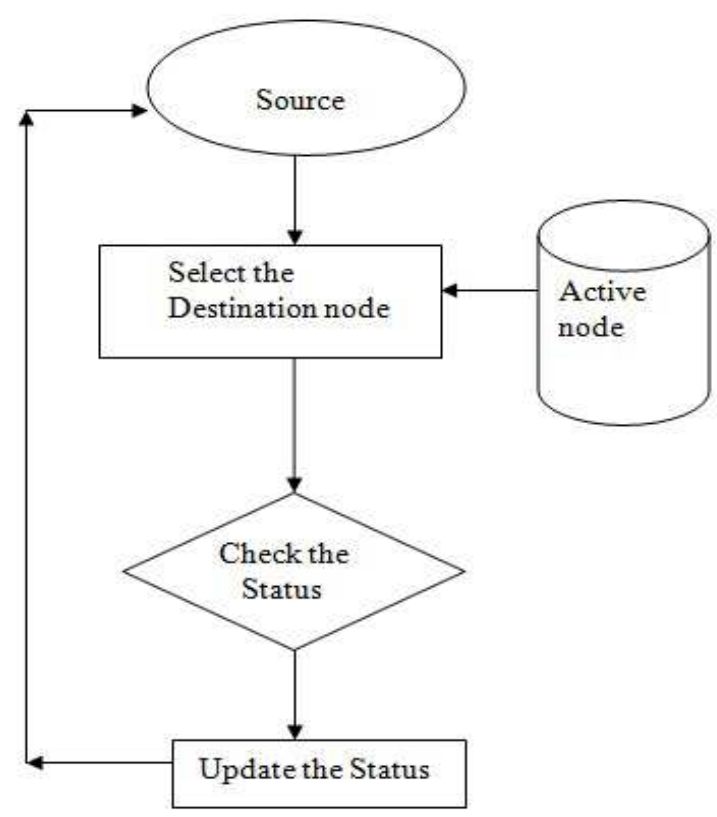

Figure 6. Change of the Status

\subsection{Schemes}

1. Basic

2. Dynamic Channel Allocation (DCA)

3. Enhance Dynamic Channel Allocation (EDCA) 


\subsubsection{Basic}

Let us assume one MBS service in the MBS network, and assume that there are $\mathrm{K}$ cells in $Z$, and $Z=\{$ Cell1, Cell2... CellK-1, Cells $\}$. Suppose that an MS currently resides in Cells. For $Z$, the variable zone is maintained in the MBSC. The zone $=$ enabled ( $z o n e=$ disabled) indicates that the MBS zone technology is enabled (disabled) in Z. Scheme Basic consists of six procedures: Service Flow Creation (SFC), Service Flow Deletion (SFD), Procedure Service Join (PSJ), Procedure Service Leave (PSL), intra-MBS zone handoff, and inter-MBS zone handoff.

To make a traditional call, an MS performs Service Flow Creation (SFC) with Cells to obtain a dedicated channel. If there is an idle dedicated channel in Cells, the new traditional call is accepted. Otherwise, the new traditional call is blocked, which is referred as "new traditional call blocking". To complete a traditional call, the MS performs Services Flow Deletion (SFD) with Cells, and a dedicated channel is released.

To join the MBS service, the MS executes Procedure Service Join (PSJ) with Cells and the MBS Controller (MBSC) to obtain a common channel. For a new MBS call arrival, the MBSC checks whether the data flow to $Z$ for the MBS service exists. If the data flow does not exist, the MBSC negotiates with all cells in Z for bearer resources, and establishes the MBS data flow to all cells in Z. Then, the MBSC sets zone to enable. All cells in Z turn on the same common channel to activate the MBS zone technology. Afterward, the MS can receive the MBS content from the common channel of Cells.

To disjoin the MBS service, the MS executes PSL with Cells and the MBSC. When an MBS call is completed, the MBSC determines whether to deactivate the MBS zone technology in Z. If there is no MBS calls in Z, the MBSC informs all cells in Z to turn off the same common channel to deactivate the MBS zone technology. The MBSC terminates the MBS data flow to $Z$, and sets zone to disabled.

During a traditional call or an MBS call, an MS may move from the old cell Cello to the new cell Celln, which is referred as "handoff". The MS performs the MAC Layer Handoff procedure to synchronize the downlink of Celln. For a handoff traditional call, the MS performs SFC with Celln to re-establish the traditional call in Celln. If an idle dedicated channel in Celln is available, the handoff traditional call request is accepted. Otherwise, the handoff traditional call is forced to terminate in Celln.

Suppose that Cello and Celln belong to $\mathrm{Zo}$ and $\mathrm{Zn}$, respectively. For a handoff MBS call, the MS checks whether $Z n$ is equal to $Z o$. If $Z n \neq Z$, the MS exercises the inter- MBS zone handoff, which is executed as follows: Through Celln, the MS notifies the MBSC that it has left Zo. If there is no MBS call in Zo, the MBSC deactivates the MBS zone technology in Zo. Afterward, the MS exercises PSJ with the MBSC to resume receiving the MBS content in Celln. Otherwise (i.e., $\mathrm{Zn}=\mathrm{Zo}$ ), the same common channel is used to multicast the MBS content, and the MS exercises the intra- MBS zone handoff. The MS continues to receive the MBS content in Celln using the same common channel.

In scheme Basic, a common channel with the MBS zone technology is always reserved to serve MBS calls.

A new MBS call request to the BS or a handoff MBS call request (i.e., an ongoing MBS call moves from the old BS to the new BS belonging to the same MBS zone) can always be accepted. To summarize, scheme Basic does not take the following two issues into consideration to enable the MBS zone technology:

I) The channel pool status (to enable the MBS zone technology, e.g., the number of served MBS calls);

II) The common channel or the dedicated channel (to serve the MBS calls).

\subsubsection{Dynamic Channel Allocation and Enhance Dynamic Channel Allocation:}

DCA and Enhanced DCA (EDCA), for more flexible channel allocation in the MBS network. In DCA, the MBS network determines whether the MBS zone technology should be enabled or disabled when an MS joins/leaves the MBS service. Scheme EDCA enhances DCA by also considering the behavior of the traditional call.

In DCA, the MBSC attempts to activate the MBS zone technology if the number of MBS calls in all cells (belonging to the same MBS zone) is equal or larger than a pre-defined $\theta$. When the MBS zone technology is disabled, DCA turns on a common channel to serve the MBS calls if the number of MBS calls in the cell is larger than dc. The EDCA enhances DCA by putting the behavior of the traditional call into consideration. When a traditional call arrives in a cell where

JTI Vol. 1, No. 1, March 2013: $26-35$ 
there is no free dedicated channel, EDCA attempts to have more dedicated channels by activating or deactivating the MBS zone technology.

- $n t, i$ : For Celli $\in Z$, the $n t, i$ counter is maintained in Celli to count the number of traditional calls in Celli.

- $n m$, i: For each Celli $\in Z$, the MBSC maintains the $n m$, i counter to count the number of MBS calls in Celli.

- $\Theta$ : The threshold $\theta$ (where $0 \leq \theta \leq d z+1$ ) is defined to control activating or deactivating the MBS zone technology.

- comi: This variable is maintained in Celli (belonging to $Z$ ) to indicate whether the common channel is turned on to serve the MBS calls, where comi = on (comi = off) implies the common channel is turned on (off). The comi variable is referenced when the MBS zone technology is disabled (i.e., zone $=$ disabled). If zone $=$ enabled, there are $\mathrm{C}^{*} \mathrm{i}=\mathrm{Ci}-\mathrm{dz}-1$ dedicated channels in Celli. Otherwise (i.e., zone $=$ disabled), there are $\mathrm{C}^{*} \mathrm{i}=\mathrm{Ci}$ and $\mathrm{C}^{*} \mathrm{i}=$ $\mathrm{Ci}-\mathrm{dc}-1$ dedicated channels in Celli when $\mathbf{c o m i}=$ off and $\operatorname{comi}=$ on, respectively.

\section{Scheme DCA:}

The DCA handles a new or handoff traditional call request in the same way of Basic. In DCA, we modify the PSJ and PSL procedures in Basic as follows:

Figure 7 and Figure 8 depicts the flowchart for PSJ_D and PSL_D. For a new MBS call arrival, the MBSC checks the zone variable (see Step 1). If zone = enabled, the MBSC sets $\mathrm{nm}, \mathrm{s} \leftarrow \mathrm{nm}, \mathrm{s}+1$ and the MBS call is served by the common channel with the MBS zone technology (see Step 2). Otherwise (i.e., zone = disabled), the MBSC negotiates with each Celli in Z for obtaining $\mathrm{Ci}$ and $\mathrm{nt}$, i (see Step 3).

Consider the following conditions:

Cond 1 : For $\mathrm{i}=1,2, \ldots, \mathrm{K}-1, \mathrm{~nm}, \mathrm{i} \geq \theta$ and $\mathrm{nt}, \mathrm{i} \leq \mathrm{Ci}-\mathrm{dz}-1$. (1)

Cond 2: $\mathrm{nm}, \mathrm{s}+1 \geq \theta$ and $\mathrm{nt}, \mathrm{s} \leq \mathrm{Cs}-\mathrm{dz}-1$. (2)

Cond 3: There exists a Celli in $\mathrm{Z}$ such that $\mathrm{nm}, \mathrm{i}<\theta$. (3)

Cond 4: For all Celli in $Z,(n m, i \leq d c$ and $n t, i+n m, i \leq C i)$, or $(n m, i>d c$ and $n t, i \leq C i-d c-1)$. (4)

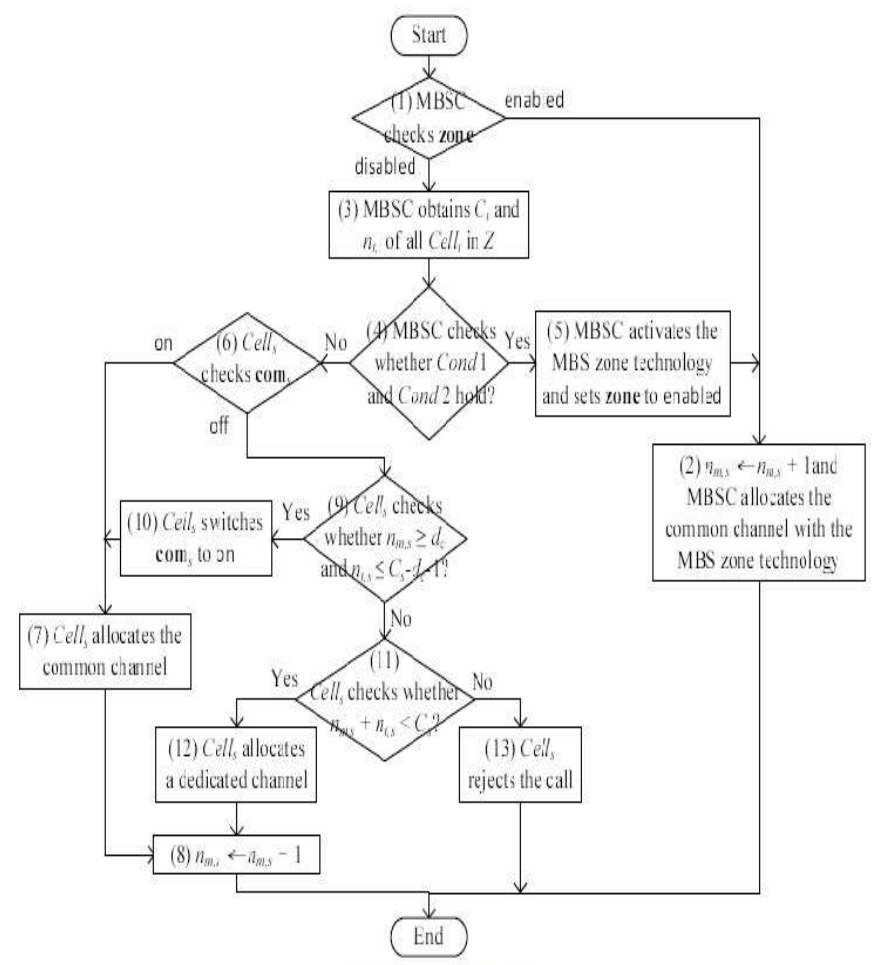

Figure 7. Flowchart for PSJ_D 


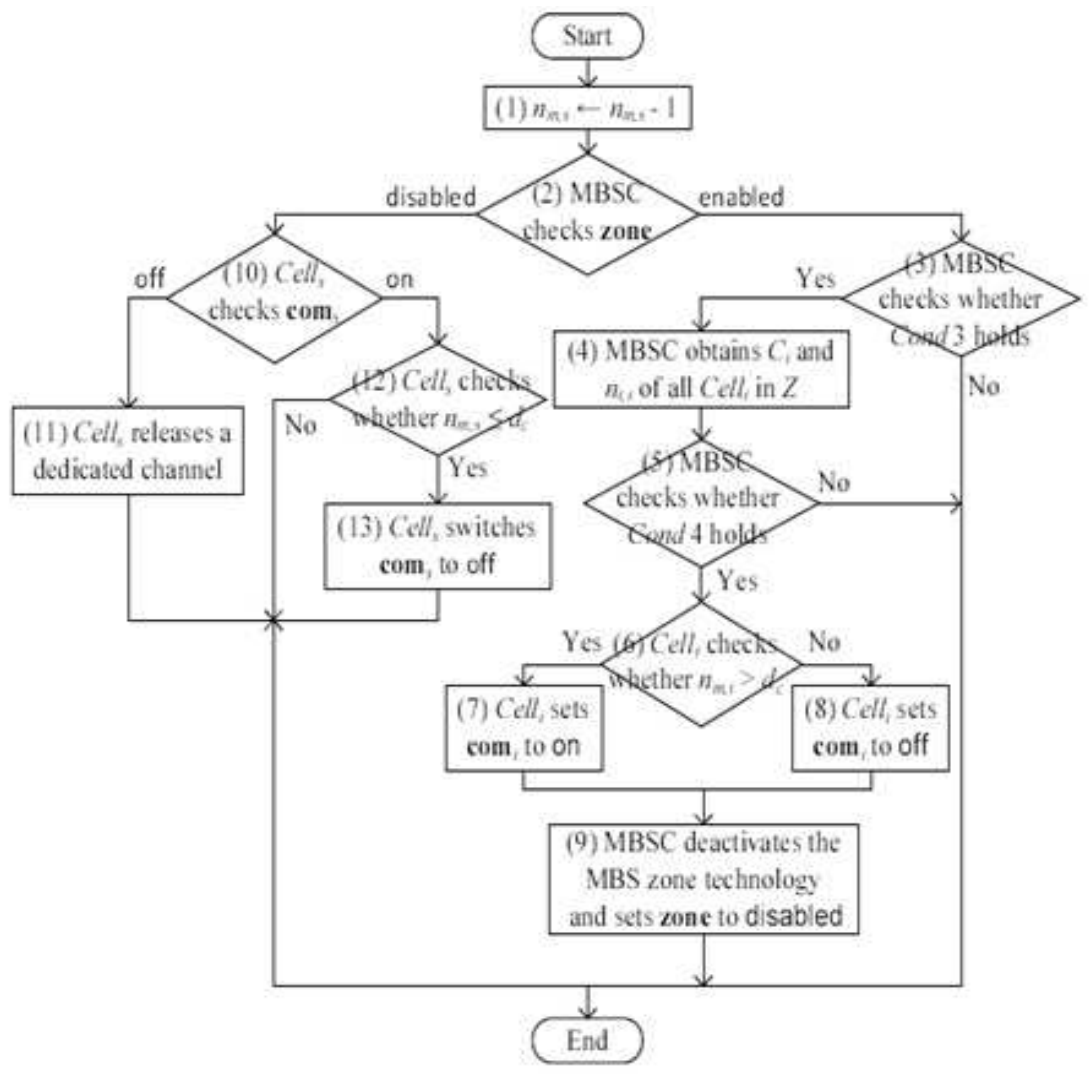

Figure 8. Flowchart for PSL_D

\section{Scheme EDCA:}

Scheme EDCA handles the new or handoff MBS call request in the same way of DCA. In EDCA, we enhances the SFC procedure, where is denoted as SFC E: For a new traditional call arrival, if there is an idle dedicated channel at Cells, the traditional call is served by the dedicated channel, and Cells sets nt,s $\leftarrow$ nt,s +1 . Otherwise, Cells informs the MBSC to obtain more dedicated channels (by activating or deactivating the MBS zone technology) to serve this traditional call request by negotiating with each Celli in $\mathrm{Z}$ for obtaining $\mathrm{Ci}$ and $\mathrm{nt}, \mathrm{i}$, and checks the zone value. Two cases are considered:

Case E1: zone = disabled. The MBSC checks the following two conditions:

Cond 5 : For $\mathrm{i}=1, \ldots, \mathrm{K}-1, \mathrm{nt}, \mathrm{i} \leq \mathrm{Ci}-\mathrm{dz}-1$. (5)

Cond 6: nt,s $+1 \leq \mathrm{Ci}-\mathrm{dz}-1$. (6)

If (5) and (6) hold, the MBSC activates the MBS zone technology. Otherwise (i.e., (5) or (6) does not hold), the MBSC does not to activate the MBS zone technology.

Case E2: zone = enabled. The MBSC checks the following two conditions:

Cond 7: For $1 \leq \mathrm{i}<\mathrm{K},(\mathrm{nm}, \mathrm{i} \leq \mathrm{dc}$ and $\mathrm{nt}, \mathrm{i}+\mathrm{nm}, \mathrm{i} \leq \mathrm{Ci})$, or $(\mathrm{nm}, \mathrm{i}>\mathrm{dc}$ and $n t, \mathrm{i} \leq \mathrm{Ci}-\mathrm{dc}-1)$. (7)

Cond 8: (nm,s $\leq \mathrm{dc}$ and $\mathrm{nt}, \mathrm{s}+\mathrm{nm}, \mathrm{s}+1 \leq \mathrm{Cs})$, or (nm,s $>\mathrm{dc}$ and $\mathrm{nt}, \mathrm{s}+1 \leq \mathrm{Cs}-\mathrm{dc}-1)$. (8)

If (7) and (8) hold, the MBSC deactivates the MBS zone technology. Otherwise (i.e., (7) or (8) does not hold), the MBSC does not to deactivate the MBS zone technology. Afterward, Cells checks whether there is idle dedicated channel. If so, the traditional call is served by the dedicated channel, and Cells sets $n t, s \leftarrow n t, s+1$. Otherwise, Cells rejects the traditional call. For a handoff traditional call (moving from Cello to Celln), the MS performs the MAC Layer Handoff procedure to synchronize the downlink of Celln. Cello sets nt,o $\leftarrow$ nt, $0-1$, and the MS performs the SFC_E procedure in Celln. Selected channel histogram is shown in Fig. 9. 


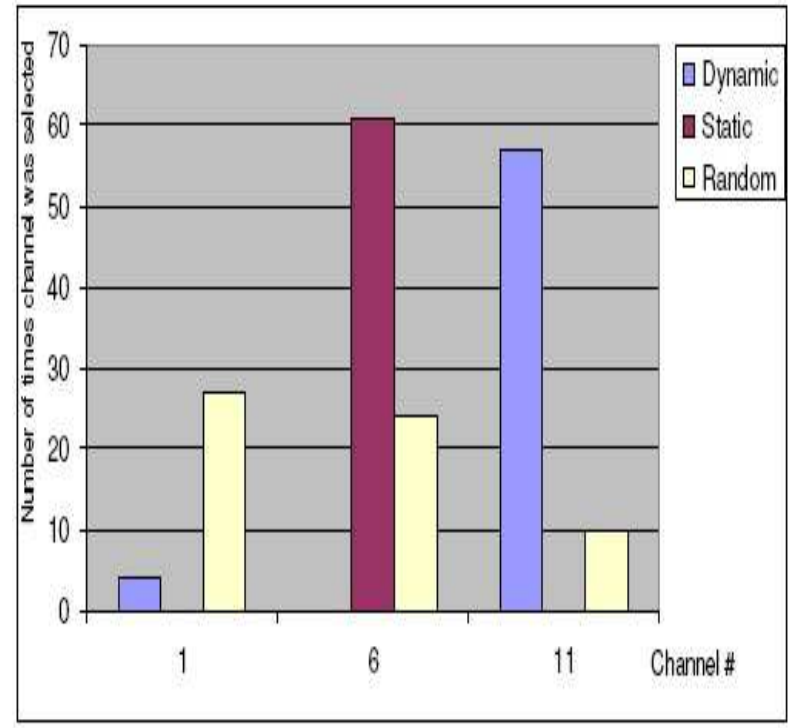

Selected channel histogram

Figure 9. Selected channel Histogram.

\section{Comparisons of DCA, and EDCA:}

In DCA, the activation of the common channel with/without the MBS zone technology is determined after the MBSC checks the channel status of all cells in an MBS zone, which introduces signalling message exchange overhead. Similar to DCA, not only for the activation of the common channel but also for the traditional call requests, EDCA introduces higher signalling message exchange overhead than DCA does. DCA and EDCA may have better system capacity, but more signalling overhead and comparison is shown in Fig. 10.

\section{Comparsion of DCA and EDCA}

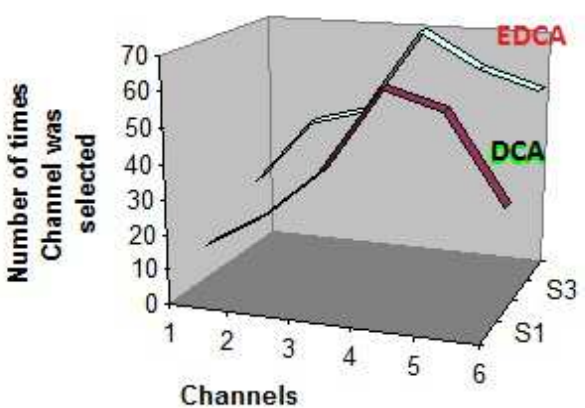

Figure 10. Comparison between DCA and EDCA.

\section{Conclusion}

This project proposed a fully developed an adaptive programming model for faulttolerant distributed computing. The underlying system model is hybrid, comprised of a synchronous part and an asynchronous part. However, such a composition can vary over time in such a way that the system may become totally synchronous or totally asynchronous.

The programming model is given by three sets (uncertain, live, and down). In this project DCA and EDCA for more flexible channel allocation for MBS, which improve the system capacity and reduce the handoff delay for the wireless MBS calls. However, more signalling overheads are caused by DCA and EDCA. Our study provides the following guidance for MBS network operators when to use Basic, DCA, or EDCA: 
$>$ When the macro diversity functions well (i.e., $d z$ is small), Basic is the good choice. On the other hand, when $d z$ is large, EDCA is the better choice.

$>$ As the number of MBS calls in a cell is larger, EDCA is the better choice.

> The performance trend for the three schemes for different MS mobility patterns are almost the same.

\section{References}

[1] IEEE. IEEE Standard for Local and Metropolitan Area Networks Part 16: Air Interface for Broadband Wireless Access Systems. IEEE Std 802.16-2009, May 2009.

[2] IEEE. IEEE 802. I 6m System Description Document. IEEE Std 802.16m, December 2008.

[3] WiMAX Forum. WiMAX Forum Network Architecture Release 1.5 Version I Draft 0 - Stage 3: Detailed Protocols and Procedure. November 2009.

[4] Wang J, Venkatachalam M, and Fang. Y System Architecture And Cross-Layer Optimization of Video Broadcast over WiMAX. IEEE Journal on Selected Areas in Communications. 2007; 25(4): 712-721.

[5] Jeng JY and Lin Y-B. Equal Resource Sharing Scheduling for PCS Data Services. ACM Wireless Networks. 1999; 5(1): 41-55.

[6] Lin YB and Yang SR. A Mobility Management Strategy for GPRS. IEEE Transactions on Wireless Communications. 2003; 2(6): 1178-1188.

[7] Lin P and Lin YB. Channel Allocation for GPRS. IEEE Transactions on Vehicular Technology. 20-01; 50(2): 375-387.

[8] Lin P. Channel Allocation for GPRS with Buffering Mechanisms. ACM Wireless Networks. 2003; 9(5): 431-441.

[9] Etemad K and Wang L. Multicast and Broadcast Multimedia Services in Mobile WiMAX Networks. IEEE Communications Magazine. 2009; 47(10): 84--91.

[10] Lai yc, Lin P, Fang Y, and Chen WH. Channel Allocation For UMTS Multimedia Broadcasting and Multicasting. IEEE Transactions on Wireless Communications. 2008; 7(11): 4375-4383.

[11] Kwon H and Lee BG. Cooperative Power Allocation for Broadcast V Multicast Services in Cellular OFDM Systems. IEEE Transactions on Communications. 2009; 57(10): 3092-3102.

[12] Zhang Y, Zhang C, Cosmas J, Loo KK, Owens T, Di Bari R, Lostanlen Y and Bard M. Analysis of DVB-H Network Coverage With the Application of Transmit Diversity. IEEE Transactions on Broadcasting. 2008; 54(3): 568-577.

[13] Rappaport SS and Hu LR. Microcellular Communication Systems with Hierarchical Macrocell Overlays: Traffic Performance Models and Analysis. Proceedings of the IEEE. 1994; 82(9): 13831397. 